\title{
Is saturated fat intake associated with CVD risk, arterial stiffness or disease severity in patients with rheumatoid arthritis?
}

\author{
L. D. Kwape ${ }^{1}$, M. A. Crilly ${ }^{2}$, J. Kyle ${ }^{1}$, N. Sarwar ${ }^{1}$, L. F. Masson ${ }^{1}$ and G. McNeill ${ }^{1}$ \\ ${ }^{1}$ Public Health Nutrition Research Group and ${ }^{2}$ Institute of Applied Health Sciences, University of Aberdeen, Foresterhill, \\ Aberdeen AB25 2ZD, UK
}

Diets high in saturated fat are associated with increased risk of CVD in the general population ${ }^{(1)}$. In patients with rheumatoid arthritis it has been suggested that diets low in fat and animal foods ${ }^{(2)}$ and high in cereals, fruit and vegetables and legumes ${ }^{(3)}$ may have beneficial effects on symptoms, although the evidence for this effect is limited. To explore the possible role of diet 114 patients with rheumatoid arthritis, but free of CVD, attending the rheumatology outpatient clinic at Aberdeen Royal Infirmary were recruited by research nurses. Standardised measurements included blood pressure, BMI and fasting blood lipid levels. Disease severity was assessed by Stanford health assessment questionnaire (HAQ) score ${ }^{(4)}$; smoking habits and alcohol consumption were assessed by self-completed questionnaires and habitual diet was assessed by the Scottish Collaborative Group FFQ version $6.5^{(5)}$. Arterial stiffness was measured by the augmentation index derived from pulse wave analysis measured using the Sphygmocor device (AtCor Medical Pty Ltd, Sydney, NSW, Australia).

Seventy-seven (68\%) of the patients completed and returned the FFQ. Median energy intake was 8.4 (interquartile range (IQR) 6.810.7) MJ/d and SFA intake was 28.2 (IQR 20.9-36.4) g/d or 12.4 (IQR 10.4-14.2) \% energy. The median Stanford HAQ score was 1.13 (IQR 0.50-1.81). Other characteristics are shown in the Table:

\begin{tabular}{|c|c|c|c|c|c|c|}
\hline & \multicolumn{2}{|c|}{ All $(n 77)$} & \multicolumn{2}{|c|}{ Male $(n$ 12) } & \multicolumn{2}{|c|}{ Female $(n 65)$} \\
\hline & Mean & SD & Mean & SD & Mean & SD \\
\hline Age (years) & 54.1 & 6.36 & 56.5 & 6.91 & 53.7 & 6.33 \\
\hline SBP $(\mathrm{mmHg})$ & 126 & 14.6 & 129 & 18.1 & 125 & 14.0 \\
\hline DBP (mmHg) & 82.3 & 9.25 & 81.6 & 7.85 & 82.4 & 9.52 \\
\hline BMI $\left(\mathrm{kg} / \mathrm{m}^{2)}\right.$ & 26.6 & 5.32 & 26.5 & 3.57 & 26.6 & 5.61 \\
\hline Total chol $(\mathrm{mmol} / \mathrm{l})$ & 5.32 & 1.19 & 5.00 & 0.96 & 5.38 & 1.23 \\
\hline HDL-chol (mmol/l) & 1.55 & 0.37 & 1.25 & 0.28 & 1.60 & 0.36 \\
\hline LDL-chol $(\mathrm{mmol} / \mathrm{l})$ & 3.28 & 0.99 & 3.13 & 0.66 & 3.31 & 1.04 \\
\hline TAG $(\mathrm{mmol} / \mathrm{l})$ & 1.09 & 0.59 & 1.32 & 0.80 & 1.05 & 0.53 \\
\hline AIX@75* & 32.5 & 7.58 & 26.7 & 8.14 & 33.2 & 7.04 \\
\hline
\end{tabular}

SBP, systolic blood pressure; DBP, diastolic blood pressure; chol, cholesterol. *Augmentation index adjusted to a heart rate of 75 beats per min.

The association between SFA intake and blood lipids, arterial stiffness and disease severity was assessed by regression analysis with adjustment for age, gender, number of years smoking, systolic blood pressure and alcohol consumption. In the fully-adjusted models the SFA intake ( $\%$ energy) was positively associated with fasting total cholesterol levels $(\beta 0.14$ (95\% CI $0.04,0.34)$ ), but there was no significant association with SFA and arterial stiffness ( $\beta 0.02(95 \%$ CI $-0.57,0.61)$ ) or disease severity $(\beta-0.03(95 \%$ CI -0.10 , $0.03)$ ).

The results suggest that reduction in dietary saturated fat may improve lipid risk factors for CVD in patients with rheumatoid arthritis, but provide no evidence that this diet would improve arterial stiffness or disease severity.

1. Hu F, Stampfer MJ, Manson JE et al. (1997) New Engl J Med 337, 1491-1499.

2. McDougall J, Bruce B, Spiller G et al. (2002) J Altern Complement Med. 8, 71-75.

3. Sköldstam L, Hagfors L \& Johansson G (2003) Ann Rheum Dis 62, 208-214.

4. Fries JF, Spitz P, Kraines RG et al. (1980) Arthritis Rheum 23, 137-145.

5. Scottish Collaborative Group (2000) Scottish Collaborative Group food frequency questionnaire. http://www.foodfrequency.org.uk/ 